\title{
Hemodynamic and Respiratory Effects of Regadenoson During Radiologic Imaging in Infants and Children
}

\author{
Brian Schloss ${ }^{\mathrm{a}, \mathrm{b}, \mathrm{d}}$, Ismail Bekiroglu ${ }^{\mathrm{a}}$, Colin O’Connor ${ }^{\mathrm{a}}$, Simon Lee ${ }^{\mathrm{c}}$, Julie Rice ${ }^{\mathrm{a}}$, \\ Stephani S. Kim ${ }^{\mathrm{a}, \mathrm{b}}$, Joseph D. Tobias ${ }^{\mathrm{b}, \mathrm{c}}$
}

\begin{abstract}
Background: Myocardial perfusion imaging using radionuclides is a well-validated, noninvasive method to aid in the diagnosis of patients with suspected or known myocardial ischemia. To increase the sensitivity of the technique, pharmacologic agents which induce coronary vasodilatation are administered. Regadenoson is a novel selective A2A receptor agonist that has similar efficacy to adenosine for cardiac magnetic resonance imaging (MRI) with a more favorable adverse effect profile and is the most widely used pharmacologic stress agent. While widely used in adults, there is limited experience with it in pediatrics, particularly young children.
\end{abstract}

Methods: The current study retrospectively reviews our experience with stress cardiac MRI using regadenoson in children requiring general anesthesia. The study cohort included eight patients, all male, ranging in age from 2 to 6.2 years (mean age of 4.2 years) and in weight from 10 to $30.5 \mathrm{~kg}$ (mean weight of $18.5 \mathrm{~kg}$ ). All patients received general anesthesia with endotracheal intubation and a volatile anesthetic agent.

Results: Heart rate 1 min prior to regadenoson was $99 \pm 19.2$ (mean \pm standard deviation (SD)) beats per minute. Peak heart rate was achieved at an average of 4 min post regadenoson administration with a mean heart rate of $122 \pm 15$ beats per minute. The average of the mean arterial pressure $1 \mathrm{~min}$ prior to regadenoson was $53.4 \pm 5.2 \mathrm{~mm}$ $\mathrm{Hg}$. Mean arterial pressure nadir was noted at $6 \mathrm{~min}$ post regadenoson with a value of $44.1 \pm 6.3 \mathrm{~mm} \mathrm{Hg}$. Blood pressure support with phenylephrine was required in four of the eight $(50 \%)$ of patients. No adverse respiratory events were noted. Only one of the eight $(13 \%)$

Manuscript submitted September 15, 2021, accepted November 29, 2021 Published online December 8, 2021

aDepartment of Anesthesiology \& Pain Medicine, Nationwide Children's Hospital, Columbus, $\mathrm{OH}, \mathrm{USA}$

${ }^{b}$ Department of Anesthesiology \& Pain Medicine, The Ohio State University College of Medicine, Columbus, OH, USA

'Division of Pediatric Cardiology, Department of Pediatrics, Nationwide Children's Hospital and The Ohio State University College of Medicine, Columbus, OH, USA

${ }^{\mathrm{d} C o r r e s p o n d i n g ~ A u t h o r: ~ B r i a n ~ S c h l o s s, ~ D e p a r t m e n t ~ o f ~ A n e s t h e s i o l o g y ~ \& ~ P a i n ~}$ Medicine, Nationwide Children's Hospital, 700 Children's Drive, Columbus, OH 43205, USA. Email: Brian.Schloss@Nationwidechildrens.org

doi: https://doi.org/10.14740/cr1323 patients had a perfusion defect but had preserved ventricular function and recovered without incident.

Conclusions: Use of regadenoson in pediatric patients requiring general anesthesia is safe and feasible.

Keywords: Pediatric anesthesia; Regadenoson; Myocardial perfusion imaging

\section{Introduction}

Various congenital, post-surgical or acquired conditions can result in disruption of the normal pattern of coronary perfusion leaving patients at risk for myocardial perfusion defects and ischemia. Although the gold standard for identifying such issues remains coronary arteriography, the procedure is invasive with potential risks. Myocardial perfusion imaging using radionuclides and exercise stress is a well-validated, noninvasive method to aid in the diagnosis of patients with suspected or known coronary perfusion concerns [1-4]. To further increase the sensitivity of the technique, pharmacologic agents are frequently administered to augment coronary vasodilatation, induce hyperemia, and identify areas of fixed stenosis or ischemia. Adenosine, dipyridamole, and dobutamine have been the pharmacological stress agents of choice for stress cardiac magnetic resonance imaging (MRI) [5-7]. However, these medications are associated with mild adverse effects including nausea, chest pain, and dizziness, and more serious side effects, such as bronchospasm and atrioventricular block. Adenosine also requires a continuous infusion with placement of two intravenous catheters, creating some logistical difficulties. Further investigations and classification of the adenosine receptors have identified that the agonism at the $\mathrm{A} 1, \mathrm{~A} 2 \mathrm{~B}$, and A3 receptors are primarily responsible for adverse effects associated with these pharmacological agents, leading to development of selective A2A receptor agonists [8,9].

Regadenoson is a novel selective $\mathrm{A} 2 \mathrm{~A}$ receptor agonist that has similar efficacy to adenosine for cardiac MRI with a more favorable adverse effect profile [10-12]. Regadenoson shares the common features of the stress perfusion agent, adenosine, such as being an effective and selective coronary vasodilator with a prompt onset of action, and a longer duration of action. It has the added benefit of allowing single-dose administration, 
obviating the need for continuous infusion. Aminophylline is also commonly utilized to reverse the effects of regadenoson and improve patient tolerance [13]. When compared with adenosine, regadenoson produces similar image quality and hemodynamic responses, with improved patient satisfaction scores due to a decreased incidence and intensity of adverse effects including flushing, chest pains, and dyspnea [12]. Regadenoson is the most widely used pharmacologic stress agent for evaluation of myocardial ischemia in adults, and its use in conjunction with stress perfusion cardiac MRI has been shown to be superior in risk stratifying adults with coronary risk factors, particularly within the intermediate risk group [14]. Similar utility is seen with the pediatric population, particularly in those with congenital cardiac surgery requiring coronary manipulation or in those with Kawasaki disease $[15,16]$. To date, there are limited reports regarding the hemodynamic and respiratory effects of regadenoson in young children during general anesthesia. The current study retrospectively reviews an 8-month experience with this novel agent for stress MRI.

\section{Materials and Methods}

This retrospective review was approved by the Institutional Review Board at the Nationwide Children's Hospital (Columbus, OH, USA). The study cohort included young children, 2 - 6 years of age, who required general anesthesia during regadenoson stress cardiac MRI; and the study was conducted in compliance with the ethical standards of the responsible institution on human subjects as well as with the Helsinki Declaration.

\section{Anesthetic technique}

In general, the anesthetic technique included inhalational or intravenous induction of general anesthesia followed by the administration of rocuronium to facilitate placement of an endotracheal tube. A neuromuscular blocking agent (rocuronium) was administered to facilitate endotracheal intubation, allow for breath holding during imaging, and lessen the requirements for volatile anesthetic agents. Maintenance anesthesia included a volatile anesthetic agent in air and oxygen. Prior to the administration of regadenoson, all patients received an isotonic fluid bolus $(7.5-10 \mathrm{~mL} / \mathrm{kg})$. Regadenoson dosing included $8 \mu \mathrm{g} / \mathrm{kg}$ (maximum $400 \mu \mathrm{g}$ ), followed by reversal with intravenous aminophylline ( $2 \mathrm{mg} / \mathrm{kg}$, maximum $75 \mathrm{mg}$ ) after imaging.

\section{Data collection}

The electronic medical record was searched and patients requiring general anesthesia during stress cardiac MRI with regadenoson were identified. Demographic and patient data included age, weight, gender, and comorbid conditions including congenital or acquired cardiac disease. The dose of regadenoson and aminophylline (reversal agent) was noted. Information
Table 1. Demographic Data of the Study Cohort

\begin{tabular}{ll}
\hline Gender, male & $8(100 \%)$ \\
Age (years) & $4.3(2,6.2)$ \\
Weight $(\mathrm{kg})$ & $19.9(10,30.5)$ \\
Body mass index $\left(\mathrm{kg} / \mathrm{m}^{2}\right)$ & $15.6(12.8,23.3)$ \\
\hline
\end{tabular}

Gender is listed as the number (percentage) while the other data are listed as the median and range.

regarding intraoperative anesthetic care included anesthetic agents and doses for premedication and intraoperative care. The type of airway used (endotracheal tube or laryngeal mask airway) was noted. Hemodynamic and respiratory changes including adverse effects were identified by reviewing vital signs (heart rate and blood pressure), and respiratory parameters including oxygen saturation, end-tidal carbon dioxide, and peak inflating pressure. Vital sign data were collected every 3 min during anesthetic care. During the regadenoson stress portion of the study, vital signs were collected every 1 min. During this time, the noninvasive blood pressure cuff was set to cycle every $1 \mathrm{~min}$. To further identify adverse effects, the need for the administration of resuscitation agents used to treat bradycardia or hypotension were noted. These included anticholinergic agents, vasoactive agents such as epinephrine or phenylephrine, and medications to treat bronchospasm including albuterol were collected. Laboratory data including complete blood count, renal function, hepatic function, and coagulation function were reviewed.

Categorical variables were summarized as numbers and percentages. Mean, standard deviation (SD), median, and interquartile range (IQR) were calculated for continuous variables. Mean (SD) were calculated for heart rate, mean arterial pressure (MAP) systolic blood pressure, and diastolic blood pressure were calculated for the $5 \mathrm{~min}$ before and $20 \mathrm{~min}$ after regadenoson administration. All analysis was completed using SAS 9.4 (Cary, NC).

\section{Results}

The study cohort include eight patients, all male, ranging in age from 2 to 6.2 years (median age of 4.3 years) and in weight from 10 to $30.5 \mathrm{~kg}$ (median weight of $19.9 \mathrm{~kg}$ ). All of the patients had a prior history of Kawasaki disease with coronary aneurysms. One patient had multiple giant aneurysms (proximal left anterior descending and right coronary artery); the other seven had small aneurysms. All patients had normal biventricular global systolic function and were otherwise asymptomatic and obtained stress perfusion imaging as a baseline. A summary of the demographic data is listed in Table 1 and anesthetic data in Table 2.

All patients received general anesthesia with endotracheal intubation following intravenous or inhalation induction. To facilitate endotracheal intubation and lessen the requirements for volatile anesthetic agents, neuromuscular blockade was provided by rocuronium $(0.6-1 \mathrm{mg} / \mathrm{kg})$. Repeat doses of rocuronium were given to five $(62.5 \%)$ patients to facilitate breath 
Table 2. Anesthetic Data of the Study Cohort

\begin{tabular}{|lll}
\hline Variable & $\begin{array}{l}\text { Number } \\
(\%)\end{array}$ & $\begin{array}{l}\text { Mean } \pm \\
\text { SD }\end{array}$ \\
\hline $\begin{array}{l}\text { Total anesthesia time (min) } \\
\text { Premedication }\end{array}$ & $98.6 \pm 7.3$ \\
$\quad$ Oral midazolam (mg) & \multicolumn{2}{c}{$7 \pm 1.7$} \\
Inhaled anesthetic agent & & \\
$\quad$ Sevoflurane & $7(87.5 \%)$ \\
$\quad$ Isoflurane & $1(12.5 \%)$ \\
Intravenous anesthetic medications & & \\
$\quad$ Propofol (mg) & $4(50 \%)$ & $25 \pm 5.8$ \\
$\quad$ Ondansetron (mg) & $7(87.5 \%)$ & $2.5 \pm 0.8$ \\
$\quad$ Dexamethasone (mg) & $4(50 \%)$ & $3.3 \pm 1.5$ \\
Airway, endotracheal tube & $8(100 \%)$ \\
Regadenoson dose ( $\mu$ g) & $8(100 \%)$ & $148 \pm 49$ \\
Aminophylline dose (mg) & $8(100 \%)$ & $4.7 \pm 1.6$ \\
Bradycardia treatment & $0(0 \%)$ & \\
Hypotension treatment, phenylephrine $(\mu \mathrm{g})$ & $4(50 \%)$ \\
\hline Dose 1 & $4(50 \%)$ & $20 \pm 14$ \\
Dose 2 & $3(37.5 \%)$ & $13 \pm 6$ \\
Dose 3 & $3(37.5 \%)$ & $15 \pm 5$ \\
\hline
\end{tabular}

SD: standard deviation.

holds during the MRI. The primary intraoperative anesthetic agent was sevoflurane in seven patients and isoflurane in one patient. Total anesthesia time varied from 89 to $108 \mathrm{~min}$ (average time of $98.6 \mathrm{~min}$ ). Intraoperative fluid administration included lactated ringers (LR) ranging from 11.4 to $24.5 \mathrm{~mL} / \mathrm{kg}$ (average $18.8 \mathrm{~mL} / \mathrm{kg}$ ). After anesthetic induction and prior to the administration of regadenoson, all patients received a bolus of $7.5-10 \mathrm{~mL} / \mathrm{kg}$ of LR. No colloid fluid was given to any patient. The stress portion of the MRI study was initiated with an intravenous regadenoson dose of $8 \mu \mathrm{g} / \mathrm{kg}$ (maximum 400 $\mu \mathrm{g})$ administered as a rapid intravenous push. Once all images were acquired, reversal of regadenoson was achieved with an intravenous aminophylline dose of $2 \mathrm{mg} / \mathrm{kg}$ (maximum 75 $\mathrm{mg}$ ), which was administered intravenously over $20-30 \mathrm{~s}$. The length of the stress test ranged from 5 to $11 \mathrm{~min}$ (average time: $9 \mathrm{~min}$ ).

Neither excessive tachycardia nor bradycardia requiring treatment or resulting in hemodynamic instability was noted. One minute prior to regadenoson, the heart rate was 99 beats per minute. Peak heart rate was achieved an average of $4 \mathrm{~min}$ after regadenoson administration with a maximum heart rate of 122 beats per minute (Fig. 1, Table 3). Six of eight patients $(75 \%)$ had a heart increase $\geq 20$ beats per minute and two patients $(25 \%)$ had a heart rate increase $\geq 30$ beats per minute. The maximum increase in heart rate was 36 beats per minute.

The average MAP 1 min prior to regadenoson was 54 $\mathrm{mm} \mathrm{Hg}$. The MAP nadir was noted at $6 \mathrm{~min}$ after regadenoson with an average low MAP of $42 \mathrm{~mm} \mathrm{Hg}$. Five of eight patients $(62.5 \%)$ had a $\geq 10 \mathrm{~mm} \mathrm{Hg}$ decrease in MAP and two patients (25\%) had a $\geq 20 \mathrm{~mm} \mathrm{Hg}$ decrease in MAP. Five of eight patients $(62 \%)$ had a MAP decrease $\geq 10 \mathrm{~mm} \mathrm{Hg}$ and two patients $(25 \%)$ had a MAP decrease $\geq 20 \mathrm{~mm} \mathrm{Hg}$. The greatest decrease in MAP was $21 \mathrm{~mm} \mathrm{Hg}$ (Table 3). Four of the eight patients received phenylephrine for hypotension during the stress portion of the study. One patient received one dose while the other three patients received three doses. No other vasoactive or anticholinergic medications were administered.

No patient experienced an oxygen desaturation event or clinical concerns for bronchospasm. Following completion of the imaging procedure, residual neuromuscular blockade was reversed with sugammadex and when awake, the patient's trachea was extubated. The patients were transported to the post-anesthesia care unit of the procedural sedation center until they had returned to their baseline status. They were then transferred to phase II recovery and discharged home per our institution's routine. No adverse events were reported in the recovery period and all patients were discharged to home on the day of the procedure.

\section{Discussion}

Regadenoson is a selective adenosine receptor agonist that acts as a coronary vasodilator. It is being used more commonly in infants and children to evaluate the coronary arteries during MRI. As was the case in our cohort of patients, these studies are performed in children at risk for or with suspected coronary ischemia related to congenital heart disease or acquired inflammatory heart disease such as Kawasaki's syndrome. In addition to causing coronary vasodilatation, as an adenosine agonist, regadenoson can lead to hemodynamic and respiratory effects including hypotension, heart block, bradycardia, and bronchospasm.

Our retrospective review showed notable decreases in MAP following regadenoson administration, with half of the patients requiring treatment with bolus doses of phenylephrine. These findings are similar to those reported previously by Wilkinson et al, Doan et al, and Noel et al [15-17]. Doan et al also noted a higher incidence of hypotension in patients undergoing stress MRI with regadenoson in patients who were sedated versus non-sedated patients $(28 \%$ versus $4 \%)$ [15]. Given the age of our patients and our routine clinical practice in the MR suite, all of our patients were receiving general anesthesia with sevoflurane via an endotracheal tube with controlled ventilation. This may have increased the incidence and magnitude of hypotension that we saw $(50 \%)$. However, in our cohort and those reported by other authors, the hypotension was easily treated with the administration of a direct acting $\alpha$-adrenergic agonist, phenylephrine. As experience with this agent increases, it may be that the administration of a more liberal isotonic bolus can be used to mitigate the hemodynamic effects of regadenoson. Others have anecdotally reported a decrease in hypotension events once they started giving a fluid bolus prior to regadenoson administration [15-17]. Our patients received a $10 \mathrm{~mL} / \mathrm{kg}$ bolus of crystalloid prior to regadenoson and we hypothesize this may have reduced the incidence and severity of hypotension requiring 
a

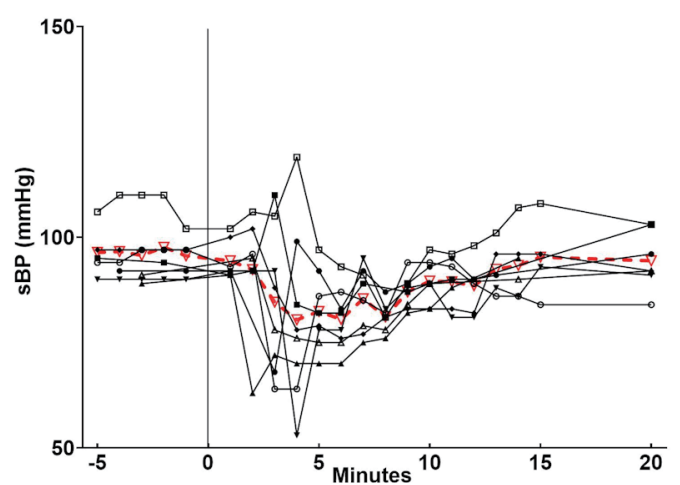

C

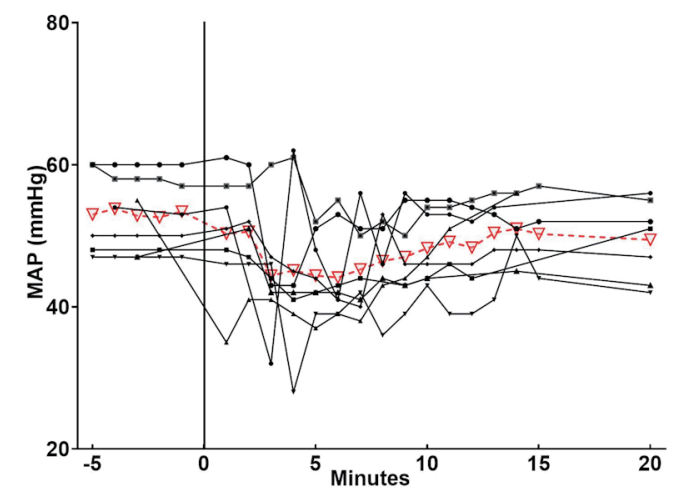

b

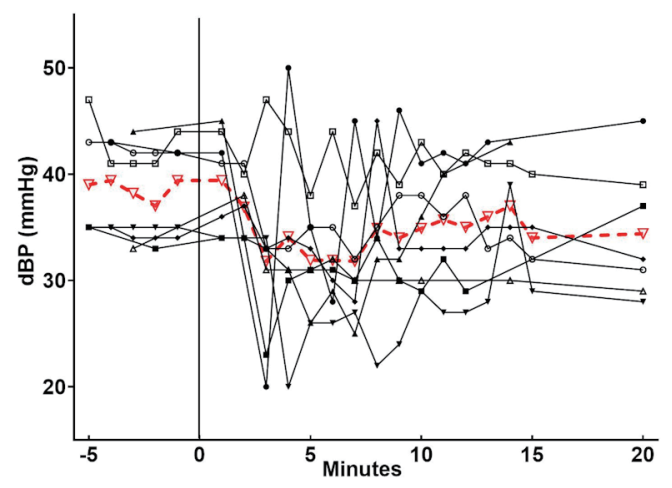

d

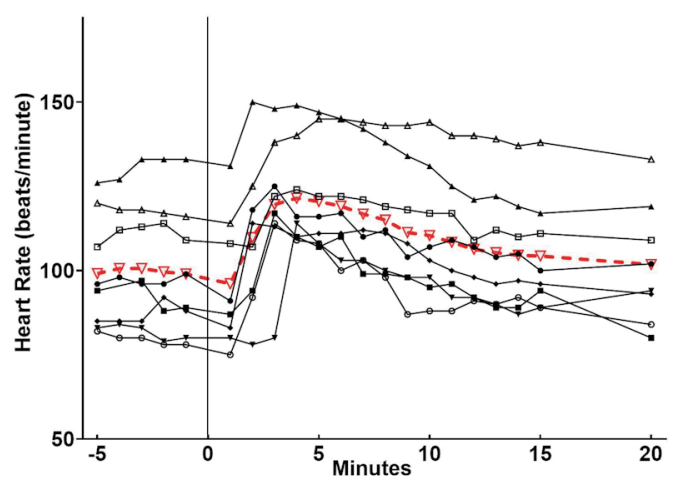

Figure 1. Heart rate and blood pressure changes following the administration of regadenoson. The individual patients are listed in black and the average in red. The administration of regadenoson occurred at time 0 . Individual patient data are listed in black with the average values in red. sBP: systolic blood pressure; dBP: diastolic blood pressure; MAP: mean arterial pressure; HR: heart rate.

pharmacologic intervention. It may be that a larger fluid bolus $(20 \mathrm{~mL} / \mathrm{kg})$ may further decrease the incidence and magnitude of hypotension. Perhaps the more dramatic and more consistent hemodynamic change that has been reported is an increase in heart rate. Previous authors have noted an increase of 30 - 50 beats per minute. In our cohort of patients, the heart increase averaged only 20 beats per minute. We would postulate that the less dramatic increase in heart rate may have been related to the concomitant administration of sevoflurane and its negative chronotropic effects. Bradycardia, heart block, sinus pauses or more concerning effects on chronotropic function were not noted.

One benefit of this dataset is the relatively homogenous anesthetic approach. The reasons for our approach include the need for general anesthesia in a younger patient population and the requirement for breath holds during specific portions of the MRI protocol. To facilitate this and ensure optimal imaging, all the patients had a similar anesthetic technique that included inhalation induction with sevoflurane in air and oxygen followed by endotracheal intubation and controlled ventilation. Propofol was administered as a single bolus dose, only at the time of induction to deepen the level of anesthesia prior to endotracheal intubation. As such, it was unlikely to have contributed to hypotension during the stress portion of the imaging. To ensure a motionless patient, facilitate breath holding sequences, and allow a decrease in volatile anesthetic requirements, a neuromuscular blocking agent (rocuronium) was administered. We specifically chose to use a neuromuscular blocking agent to minimize the amount of volatile agent required and thus minimize hypotension.

Limitations of our study included the small sample size of eight as well as the lack of detailed respiratory data during the anesthetic. Unfortunately, the difficulties of performing general anesthesia in zone 4 of an MRI suite prevent our electronic medical record from collecting ventilator data to further support the lack of respiratory effects following regadenoson. We are unable to report specific data regarding tidal volume or airway pressure changes during the stress portions of the study. However, no episodes of oxygen desaturation and no clinical concerns regarding the adequacy of ventilation were found.

Although there are ample data regarding the efficacy of regadenoson in facilitating stress MRI, there are relatively limited data regarding the physiologic effects of regadenoson in sedated infants and children [15-17]. Furthermore, the data regarding the sedation reported in these studies were heterog- 
Table 3. Hemodynamic Changes After Regadenoson

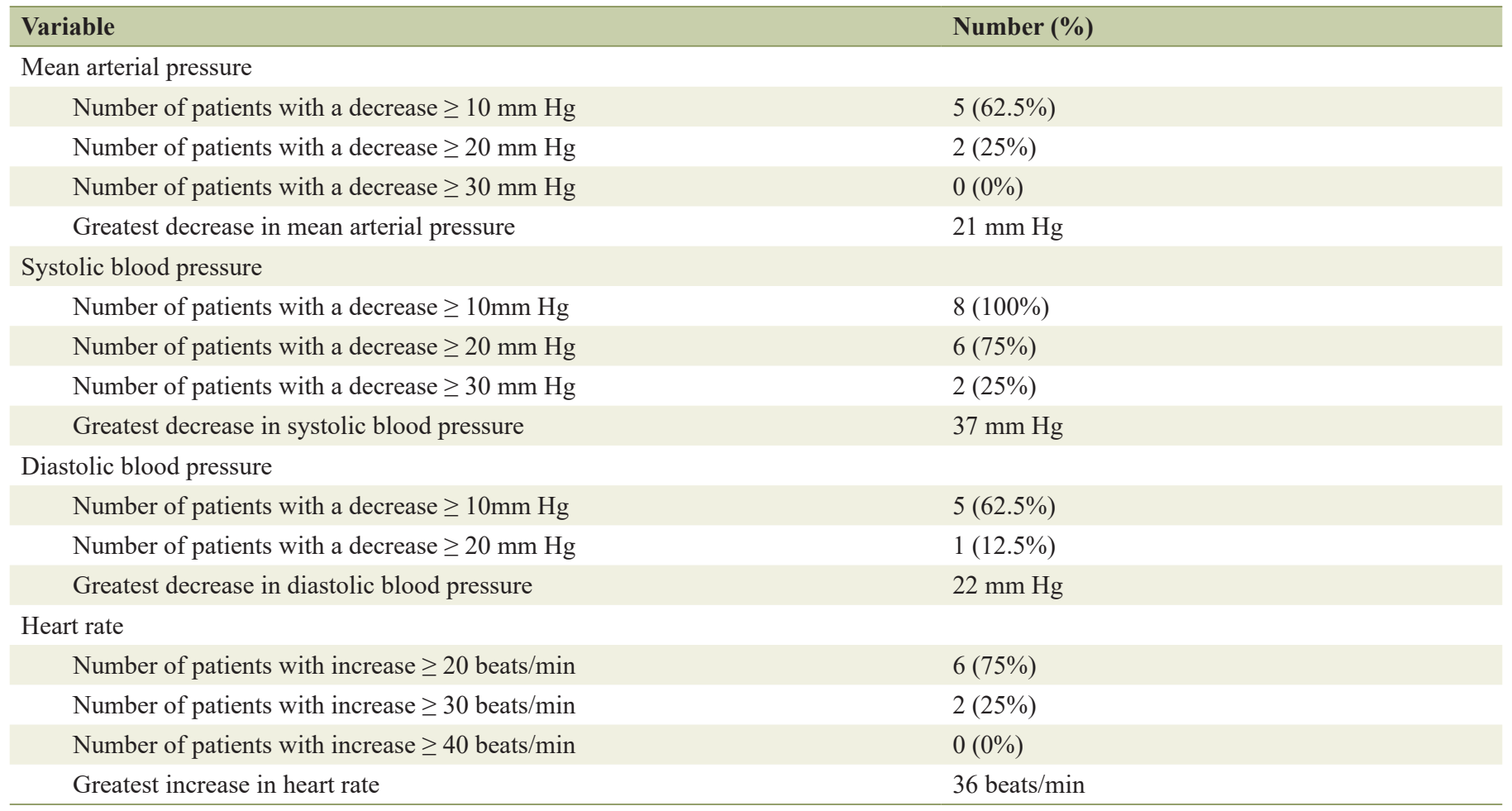

enous and without granularity. To our knowledge this is the first study to present data regarding the physiologic end-organ effects of regadenoson from the anesthesiologist's perspective with specific information regarding the technique used for general anesthesia. Based on our preliminary experience, regadenoson is generally safe to use in pediatric patients, even when administered in association with general anesthesia. We would postulate that the hemodynamic effects of regadenoson can be affected by associated sedation or general anesthetic agents. As noted previously, an increase in heart rate is commonplace, averaging 30 - 50 beats per minute in the awake state and 20 beats per minute in our cohort during general anesthesia. The incidence and magnitude of the decrease in blood pressure may be increased during the administration of a volatile anesthetic agent such as sevoflurane. However, hypotension is generally short-lived, easily treated with intermittent doses of phenylephrine, and potentially prevented by fluid loading.

\section{Acknowledgments}

We would like to thank the faculty and staff in the MRI Department at Nationwide Children's Hospital who participated in the care of these patients.

\section{Financial Disclosure}

None to declare.

\section{Conflict of Interest}

None of the authors have conflict of interest related to this manuscript.

\section{Informed Consent}

Protocol was reviewed by the hospital Institutional Review Board, and need for informed consent was not deemed necessary.

\section{Author Contributions}

Brian Schloss, Simon Lee, and Joseph D. Tobias: draft work and final approval; Ismail Bekiroglu, Colin O'Connor, and Julie Rice: acquisition of data; Stephani S. Kim: analysis of data.

\section{Data Availability}

The data supporting the findings of this study are available from the corresponding author upon reasonable request.

\section{References}

1. Prakash A, Powell AJ, Krishnamurthy R, Geva T. Mag- 
netic resonance imaging evaluation of myocardial perfusion and viability in congenital and acquired pediatric heart disease. Am J Cardiol. 2004;93(5):657-661.

2. Lederlin M, Thambo JB, Latrabe V, Corneloup O, Cochet $\mathrm{H}$, Montaudon M, Laurent F. Coronary imaging techniques with emphasis on CT and MRI. Pediatr Radiol. 2011;41(12):1516-1525.

3. Tang L, Merkle N, Schar M, Korosoglou G, Solaiyappan M, Hombach V, Stuber M. Volume-targeted and wholeheart coronary magnetic resonance angiography using an intravascular contrast agent. J Magn Reson Imaging. 2009;30(5):1191-1196.

4. Agrawal H, Wilkinson JC, Noel CV, Qureshi AM, Masand PM, Mery CM, Sexson-Tejtel SK, et al. Impaired myocardial perfusion on stress CMR correlates with invasive FFR in children with coronary anomalies. J Invasive Cardiol. 2021;33(1):E45-E51.

5. Nagel E, Lehmkuhl HB, Bocksch W, Klein C, Vogel U, Frantz E, Ellmer A, et al. Noninvasive diagnosis of ischemia-induced wall motion abnormalities with the use of high-dose dobutamine stress MRI: comparison with dobutamine stress echocardiography. Circulation. 1999;99(6):763-770.

6. Lette J, Tatum JL, Fraser S, Miller DD, Waters DD, Heller G, Stanton EB, et al. Safety of dipyridamole testing in 73,806 patients: the Multicenter Dipyridamole Safety Study. J Nucl Cardiol. 1995;2(1):3-17.

7. Voigtlander T, Schmermund A, Bramlage P, Elsasser A, Magedanz A, Kauczor HU, Mohrs OK. The adverse events and hemodynamic effects of adenosine-based cardiac MRI. Korean J Radiol. 2011;12(4):424-430.

8. Fredholm BB, AP IJ, Jacobson KA, Klotz KN, Linden J. International Union of Pharmacology. XXV. Nomenclature and classification of adenosine receptors. Pharmacol Rev. 2001;53(4):527-552.

9. Fredholm BB, Arslan G, Halldner L, Kull B, Schulte G, Wasserman W. Structure and function of adenosine recep- tors and their genes. Naunyn Schmiedebergs Arch Pharmacol. 2000;362(4-5):364-374.

10. Gao ZG, Jacobson KA. Emerging adenosine receptor agonists. Expert Opin Emerg Drugs. 2007;12(3):479-492.

11. Al Jaroudi W, Iskandrian AE. Regadenoson: a new myocardial stress agent. J Am Coll Cardiol. 2009;54(13):11231130.

12. Iskandrian AE, Bateman TM, Belardinelli L, Blackburn $\mathrm{B}$, Cerqueira MD, Hendel RC, Lieu H, et al. Adenosine versus regadenoson comparative evaluation in myocardial perfusion imaging: results of the ADVANCE phase 3 multicenter international trial. J Nucl Cardiol. 2007;14(5):645-658.

13. Rangel MO, Demori RM, Doukky R. Age and gender as predictors of benefit from aminophylline administration in patients undergoing regadenoson stress myocardial perfusion imaging: a substudy of the ASSUAGE trial. Am J Ther. 2013;20(6):622-629.

14. Antiochos P, Ge Y, Heydari B, Steel K, Bingham S, Abdullah SM, Mikolich JR, et al. Prognostic value of stress cardiac magnetic resonance in patients with known coronary artery disease. JACC Cardiovasc Imaging. 2021.

15. Doan TT, Wilkinson JC, Loar RW, Pednekar AS, Masand PM, Noel CV. Regadenoson stress perfusion cardiac magnetic resonance imaging in children with kawasaki disease and coronary artery disease. Am J Cardiol. 2019;124(7):1125-1132.

16. Noel CV, Krishnamurthy R, Masand P, Moffett B, Schlingmann T, Cheong BY, Krishnamurthy R. Myocardial stress perfusion MRI: experience in pediatric and young-adult patients following arterial switch operation utilizing regadenoson. Pediatr Cardiol. 2018;39(6):1249-1257.

17. Wilkinson JC, Doan TT, Loar RW, Pednekar AS, Trivedi PM, Masand PM, Noel CV. Myocardial stress perfusion MRI using regadenoson: a weight-based approach in infants and young children. Radiol Cardiothorac Imaging. 2019;1(4):e190061. 